\title{
Glutamyl Endopeptidase Protein Cleavage Reagent
}

National Cancer Institute

\section{Source}

National Cancer Institute. Glutamyl Endopeptidase Protein Cleavage Reagent. NCI

Thesaurus. Code C161884.

A protein cleavage reagent comprised of the bacterial serine proteinase glutamyl

endopeptidase that cleaves proteins into peptides at the C-terminal side of glutamic acid or aspartic acid residues. 